\title{
MAPPING AND CHANGE ANALYSIS IN MANGROVE FOREST BY USING LANDSAT IMAGERY
}

\author{
T. T. Dan ${ }^{a^{*}}$, C. F. Chen ${ }^{b}$, S. H. Chiang ${ }^{\text {b }}$ S. Ogawa ${ }^{a}$ \\ ${ }^{a}$ Graduate School of Engineering, Nagasaki University, Nagasaki, Japan - \\ ttdan@ctu.edu.vn,ogawa_susumu_phd@yahoo.co.jp \\ ${ }^{\mathrm{b}}$ Centre for Space and Remote Sensing Research, National Central University, Taiwan - \\ cfchen@csrsr.ncu.edu.tw, gilbert@csrsr.ncu.edu.tw
}

Commission VI, WG VI/4

KEY WORDS: Mangrove forest, Change detection, Image classification, Deforestation, Landsat data

\begin{abstract}
:
Mangrove is located in the tropical and subtropical regions and brings good services for native people. Mangrove in the world has been lost with a rapid rate. Therefore, monitoring a spatiotemporal distribution of mangrove is thus critical for natural resource management. This research objectives were: (i) to map the current extent of mangrove in the West and Central Africa and in the Sundarbans delta, and (ii) to identify change of mangrove using Landsat data. The data were processed through four main steps: (1) data pre-processing including atmospheric correction and image normalization, (2) image classification using supervised classification approach, (3) accuracy assessment for the classification results, and (4) change detection analysis. Validation was made by comparing the classification results with the ground reference data, which yielded satisfactory agreement with overall accuracy $84.1 \%$ and Kappa coefficient of 0.74 in the West and Central Africa and $83.0 \%$ and 0.73 in the Sundarbans, respectively. The result shows that mangrove areas have changed significantly. In the West and Central Africa, mangrove loss from 1988 to 2014 was approximately 16.9\%, and only $2.5 \%$ was recovered or newly planted at the same time, while the overall change of mangrove in the Sundarbans increased approximately by $900 \mathrm{~km}^{2}$ of total mangrove area. Mangrove declined due to deforestation, natural catastrophes deforestation and mangrove rehabilitation programs. The overall efforts in this study demonstrated the effectiveness of the proposed method used for investigating spatiotemporal changes of mangrove and the results could provide planners with invaluable quantitative information for sustainable management of mangrove ecosystems in these regions.
\end{abstract}

\section{INTRODUCTION}

Mangrove grows in river deltas, estuarine complexes and coasts in the tropical and subtropical regions throughout the world. It also inhabits on the shorelines and islands in sheltered coastal areas with locally variable topography and hydrology (Lugo \& Snedaker, 1974). The total mangrove area accounts for $0.7 \%$ of total tropical forests of the world. The largest extent of mangrove is found in Asia (42.0\%) followed by Africa (20.0\%), North and Central America (15.0\%), Oceania (12.0\%) and South America (11.0\%) (Giri et al., 2011). Mangrove is one of the most threatened and vulnerability ecosystems. Based on the importance and vulnerable of mangrove ecosystems faced, many studies on mangrove have been conducted to solve these issues in difference scales, long-term monitoring and detecting mangrove by using remote sensing techniques (Blasco et al., 2001; Everitt et al., 2008; Giri et al., 2007; Green, 1998; Seto \& Fragkias, 2007; and Vaiphasa et al., 2006).

The earth observation satellite data (such as Landsat) is useful for change detection applications. The distribution and abundance of mangrove in different regions of the world have been assessed with a variety of techniques. The local variability of studies spans all continents. Several studies have been carried out to investigate and compare the suitability of various classification algorithms for the spectral separation of mangrove. Change detection is a powerful tool to visualize, measure, and better to understand a trend in mangrove ecosystems. It enables the evaluation changes over a long period of time as well as the identification of sudden changes due to natural or dramatic anthropogenic impacts (for example: tsunami destruction or conversion to shrimp farms). Thus distribution, condition, and increase or decrease were the measured features used in the change-detection applications of mangrove. Monitoring change in mangrove was adopted by many researchers throughout the world (Giri et al., 2011; Giri et al., 2007; Ruiz-Luna and Berlanga-Robles, 2002; Concheddaa et al., 2008; Selvam et al., 2003; Chen et al., 2013) and the application of the supervised Maximum Likelihood Classifier (MLC) was the most effective and robust method for classifying mangroves based on traditional satellite remote sensing data.

African mangrove was widespread along the west coast from Senegal to Congo that was interlinked with highly productive coastal and tidal estuaries (UNEP, 2006). Regional conditions enabled mangrove to grow as far as 100 kilometer inland, due to strong tidal influences on rivers such as the River Gambia, the Sine-Saloum delta in Senegal, and Guinea Bissau. The overall trend for the region using area estimated from 1980 to 2006 indicated a moderate decline of mangrove covers. In the other side, mangrove in the Southeast Asia (Sundarbans delta) is located at the latitudes $21^{\circ} 30^{\prime} \mathrm{N}$ to $22^{\circ} 30 \mathrm{~N}$, and longitudes $89^{\circ} 00^{\prime} \mathrm{E}$ to $89^{\circ} 55^{\prime} \mathrm{E}$. They consist of about 200 islands, separated by about 400 interconnected tidal rivers, creeks, and canals. The Sundarbans declared as a Reserve Forest in 1875 and became the UNESCO World Heritage Site in 1999. The mangrove of the Sundarbans is dependent on natural regeneration for its existence. The most important value of the Sundarbans lies in its protective role. It helps hold coastlines, reclaim coastal lands, and settle the silt carried by rivers. For this reason, the research was adopted to detect spatial and temporal change in mangrove during the past three decades, from 1988 to 2014 in two sites of study (West and Central Africa and Sundarbans) by using a supervised classification approach.

\footnotetext{
* Corresponding author
} 


\section{MATERIALS AND METHODS}

\subsection{Study area}

The study area included mangrove areas in coastal estuaries, rivers in the West Africa (Senegal, Gambia, and Guinea Bissau) and the Sundarbans delta (Bangladesh and India) (Figure 1).

\subsubsection{West and Central Africa}

African mangrove provided these areas with essential organic nutrients as well as critical breeding grounds and nurseries for larval and juvenile stages of important fishery species. From past three decades, mangrove cover areas moderately decreased, especially in West and Central Africa (Figure 3). Rivers were dammed, their waters were diverted and the intertidal zone were extensively developed for agriculture or aquaculture, resulting in the destruction of mangrove. Large tracts of mangrove have been also converted to rice fields, fish and shrimp ponds, industrial, urban and tourism development and other non-forest used. In overpopulated and acute fuelwood-deficient areas, even small branches and saplings were removed primarily for domestic fuel. On a larger scale, salt was harvested from evaporation ponds or shallow brine filled pits, usually built in cleared mangrove areas.

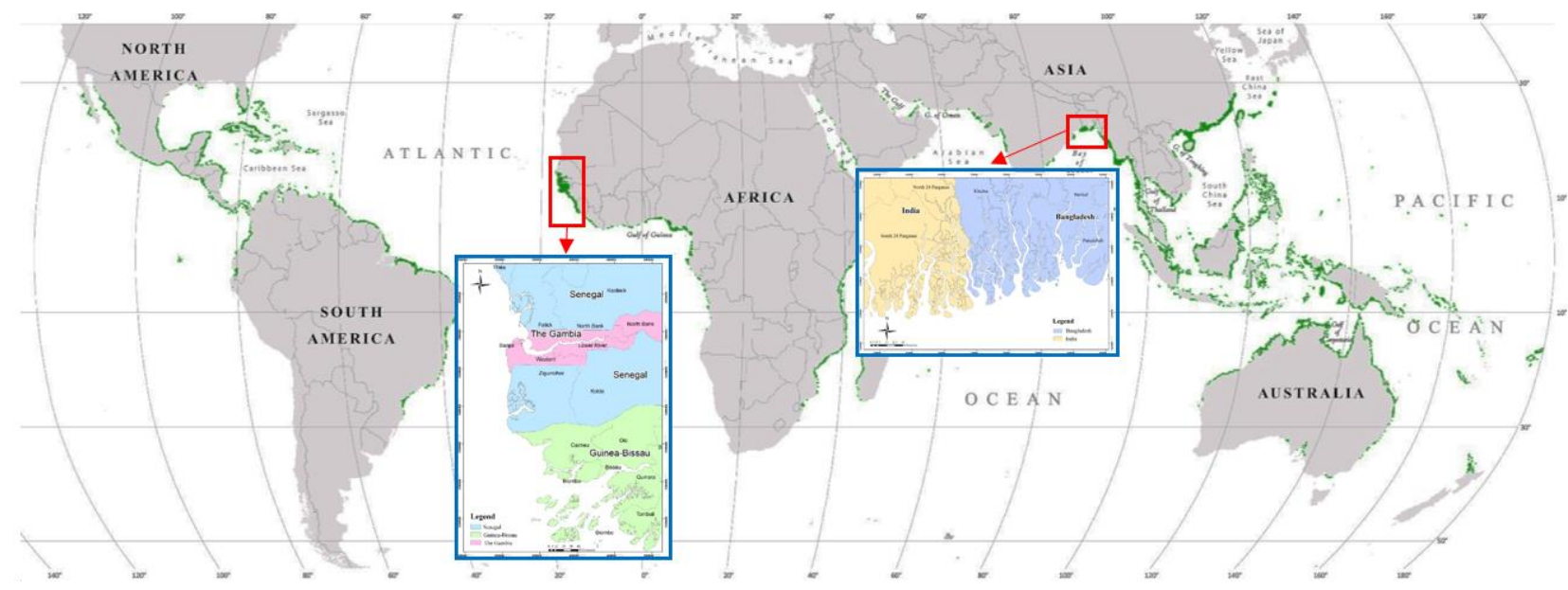

Figure 1. Two sites of study in West and Central Africa and Sundarbans delta.

\subsubsection{Sundarbans, Bangladesh and India}

The Sundarbans delta is located on both sides of the border between Bangladesh and India (Figure 4). There was the largest continuous mangrove in the world. The area height ranged from 0.9 to $2.1 \mathrm{~m}$ above the mean sea level. Mangrove was gradually increasing in area. The mangrove was located in a zone of cyclonic storms and tidal bores that originate from the Bay of Bengal and periodically strike the coastal areas. At the beginning of the colonial era (1757) in India, the Sundarbans mangrove occupied approximately twice as much as its current extent (Islam et al., 1997). To prevent further deterioration, the Bangladesh government has adopted several strategies such as the sustainable ecosystem management for both production and protection purposes.

\subsection{Data collection}

Landsat imageries in 1988, 2001, and 2014 were collected from the USGS via the website. Image acquisition date is important because vegetation and crops reflect differently at the beginning and the end of the rainy season due to phenological and temperatures disparities, and their reflectance varies from the dry season to the rainy season. The Landsat TM and ETM+ have 7 spectral bands with a spatial resolution of $30 \mathrm{~m}$ for bands 1-5 and 7. The TM and ETM+ band 6 (thermal infrared) is acquired in $120 \mathrm{~m}$ and $60 \mathrm{~m}$ resolution but is resampled to $30 \mathrm{~m}$ pixels, respectively. The Landsat 8 data have 9 spectral bands with a spatial resolution of $30 \mathrm{~m}$ for bands $1-7$ and 9 , while band 8 has a spatial resolution of $15 \mathrm{~m}$ (panchromatic band).

Moreover, DEM with a 30-m spatial resolution collected from National Aeronautics and Space Administration (NASA) was used to remove regions of elevation higher than $30 \mathrm{~m}$ and the ocean (elevation of zero). The mangrove map of USGS in 2011 was downloaded from the Ocean Data Viewer website for validating interpreted mangrove maps. In addition, this research used Google Earth and other reference maps, reports and literatures on the states of the mangrove distribution for additional information in the study area.

\subsection{Methods}

The methodology was adopted for four main steps: (1) data preprocessing including atmospheric and geometric corrections, and reflectance normalization, (2) image classification using Support Vector Machine (SVM), (3) accuracy assessment, and (4) change detection analysis.

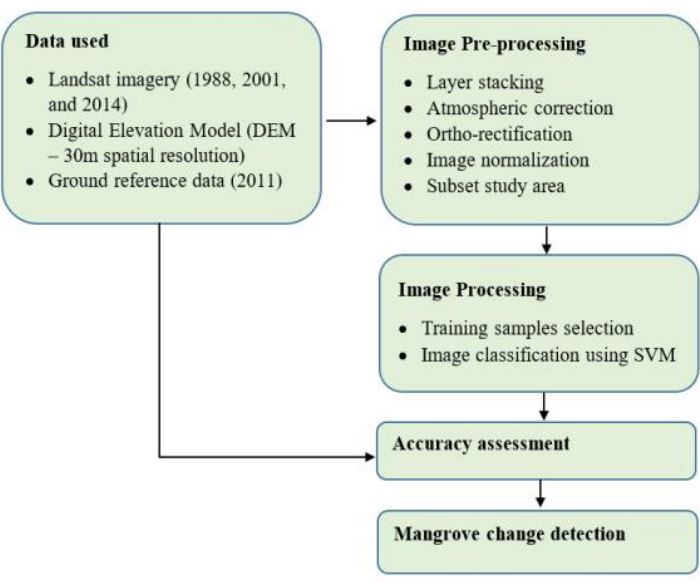

Figure 2. Flow chart of the methodology used for extract mangrove in the study area. 


\subsubsection{Image pre-processing}

This research used all spectral bands and NDVI (an additional band) to perform image classification. Remotely sensed data acquired showed some forms of distortion or shift in geometric location from one sensor to the other. Thus, image registration was necessary to fix this problem. Image registration can be defined as the transformation of an image or a map with respect to another so that the properties of any resolution elements of the object image addressable by the same coordinate pair in either one of the images (Cideciyan et al., 1992). Ground control points were used to correct geometric and a root mean square error (RMSE) of 0.58 and 0.81 pixels were obtained in the West and Central Africa and in the Sundarbans delta, respectively.

Landsat TM and ETM+ used Climate Data Records (CDR) products. The surface reflectance $\mathrm{CDR}$ generated from specialized software called Landsat Ecosystem Disturbance Adaptive Processing System (LEDAPS). The software applies MODIS atmospheric correction routines to Level-1 Landsat TM or ETM+ data. Water vapor, ozone, geopotential heights, aerosol optical thickness, and digital elevations were input with Landsat data to the Second Simulation of a Satellite Signal in the Solar Spectrum (6S) radiative transfer models to generate the top of atmosphere (TOA) reflectance, surface reflectance, brightness temperature, and to mask clouds, cloud shadows, adjacent clouds, land, and water. Therefore, the atmospheric correction only performed for Landsat 8 using Actor 2 (flat terrain, two geometric degrees-of-freedom (DOF)) software. The detailed parameters applied for the atmospheric correction presented in Table 1.

\begin{tabular}{|c|c|c|c|c|}
\hline Sensor & \multicolumn{4}{|c|}{ Landsat 8 OLI } \\
\hline \multicolumn{5}{|l|}{ West and Central Africa } \\
\hline Date & $1 / 5 / 2014$ & $1 / 5 / 2014$ & $1 / 30 / 2014$ & $1 / 30 / 2014$ \\
\hline Solar zenith angle (deg) & 37.9 & 37.9 & 37.9 & 37.9 \\
\hline Solar azimuth angle (deg) & 133.8 & 133.8 & 133.8 & 133.8 \\
\hline Water vapor category & Tropical & Tropical & Tropical & Tropical \\
\hline Aerosol type & Maritime & Maritime & Maritime & Maritime \\
\hline Average visibility (km) & 29 & 29 & 29 & 29 \\
\hline \multicolumn{5}{|c|}{ Southeast Asia - Sundarbans delta } \\
\hline Date & $1 / 29 / 2014$ & $1 / 15 / 2014$ & & \\
\hline Solar zenith angle (deg) & 35.5 & 34.9 & & \\
\hline Solar azimuth angle (deg) & 123.8 & 135.3 & & \\
\hline Water vapor category & Tropical & Tropical & & \\
\hline Aerosol type & Maritime & Maritime & & \\
\hline Average visibility $(\mathrm{km})$ & 39 & 39 & & \\
\hline
\end{tabular}

Table 1. Parameters used for atmospheric correction model.
As the results of image acquisition, the date determined image quality. They had different imageries on different dates in one period. Thus, reflectance normalization was performed with a histogram matching model which was developed by using ERDAS IMAGINE. The result of reflectance normalization is shown in Figure 3. Furthermore, two sites of the study were a very big size. It covered by two images in the Sundarbans and four images in the West and Central Africa. Hence, the subset study area was reduced the bulk and the size of information was processed. This reduced the time consumed for the analysis of satellite images and also speeded up processing due to small amount of processed data.

\subsubsection{Masking out non-vegetation and height}

Because of consideration on mangrove areas, non-vegetated areas (such as water bodies, urban, and bare land) and heights should be masked out. Non-vegetated areas were masked out by using Normalized Difference Vegetation Index (NDVI). The areas with its value less than 0.2 were masked out. This selected a threshold to check by comparing the masking results with the ancillary map, and reliable excluded for separating vegetated and non-vegetated areas in Landsat images. Furthermore, mangrove in two areas were mainly distributed in the coastal estuaries. Based on the characteristics, the distribution of mangrove and the ground reference map in 2011, mangrove was normally located in areas where the elevation was lower than $30 \mathrm{~m}$ (Chen et al., 2013). Therefore, the research was eliminated the areas higher than $30 \mathrm{~m}$ in both two sites of the study (Figure 4). In the other hand, buffer zone from the coastlines were generated. The buffer distance was different in each continental, depending the spatial distribution of mangrove on that region. In this case, $18-\mathrm{km}$, and $5-\mathrm{km}$ buffers were used for West and Central Africa and the Sundarbans delta.

\subsubsection{Image classification}

\section{Training samples selection}

From the training samples, they identified examples of land cover types of interest on the image. The image processing software system was then used to develop a statistical characterization of the reflectance each class. The image was classified by examining the reflectance each pixel and made a decision for which of the signatures it resembled the most (Eastman, 1995). For each study period, the Region of Interest (ROI) tool that provided in ENVI was used to select the training samples. Totally, there were six selected ROIs, including mangrove, cultivation, vegetation, forest, bare land and water. Each ROI represented a land cover category. 


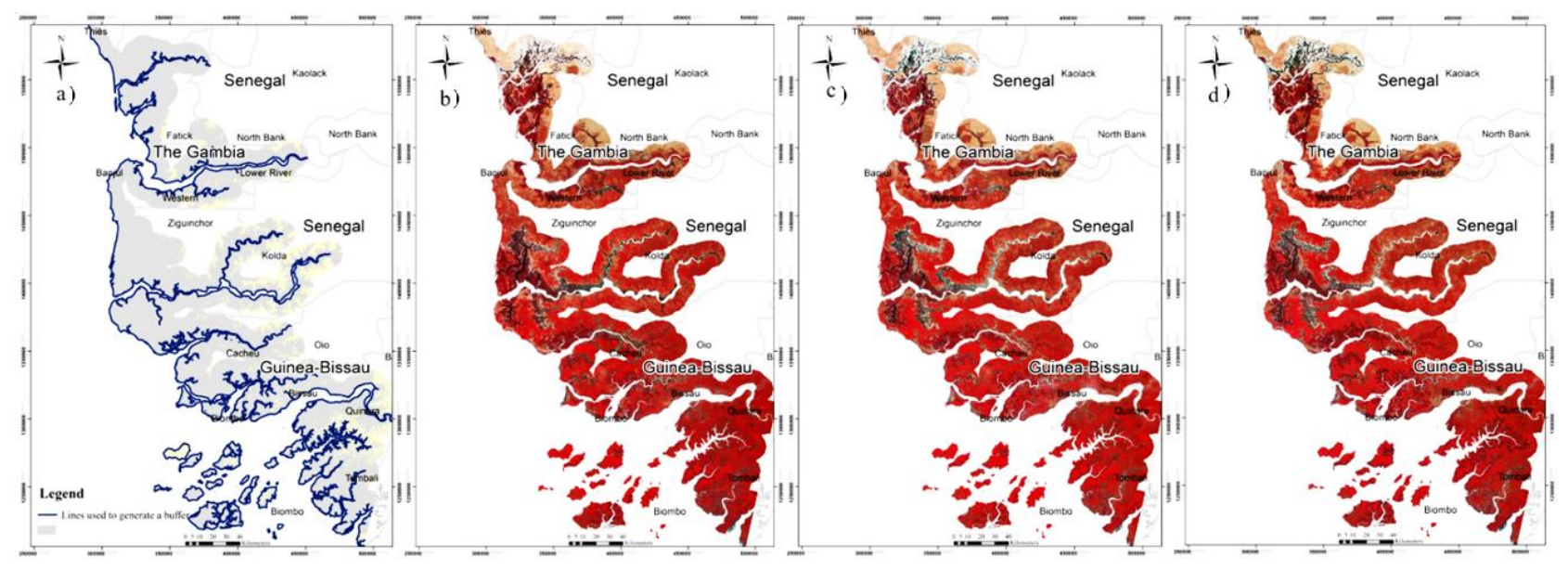

Figure 4. Study area after performing pre-processing and masking out non-vegetation area. a) Study area, b) Landsat image in 1988, c) Landsat image in 2001, and d) Landsat image in 2014 (band combination: $R=N I R, G=R, R=B$ ).

\section{Evaluation training samples}

A separability test is one of methods to determine how similar the distributions for two groups of pixels are. The JefferiesMatusita (JM) distance was a function of separability that directly related to the probability of how good a resultant classification will be (Swain et al., 1971). As the results of training data selection, they were evaluated for agreement to classify the images by using the JM from the following form:

$$
J_{i j}=\sqrt{2\left(1-e^{-\beta_{i j}}\right)}
$$

In which $\beta_{i j}$ is the Bhattacharya Distance and is given by

$$
B D=\frac{1}{8}\left[\mu_{i}-\mu_{j}\right]^{T}\left(\frac{\epsilon_{i}-\epsilon_{j}}{2}\right)^{-1}\left[\mu_{i}-\mu_{j}\right]+\frac{1}{2} \ln \left[\frac{\frac{1}{2}\left|\epsilon_{i}+\epsilon_{j}\right|}{\sqrt{\left|\epsilon_{i}\right|\left|\epsilon_{j}\right|}}\right]
$$

where $i$ and $j$ are the two signature classes,

$\mu_{i}$ is the mean vector signature for class $i$,

$\epsilon_{i}$ is corresponding class covariance matrix signature,

$T$ is the transposition function.

The JM distance had values 0 to 2 . If JM value was greater than 1.9 , then the classes show good separability. If values are between $1.7-1.9$, the separation between the classes was fairly good below 1.7, the classes were poorly separated (Jensen, 1996).

\section{Image classification}

The Support Vector Machine (SVM) algorithm was a nonparametric classifier. The method based on statistical learning theory using a kernel function to non-linearly project the training data in the input space into a higher dimensional space, where the classes were linearly separable. The SVM has been widely applied in remote sensing for classification of land uses or land cover types. It has been demonstrated to give better classification results among the maximum likelihood, univariate decision trees, and back-propagation neural networks (Huang et al., 2002). Nevertheless, it was also claimed that using the SVM for classifying high-dimensional datasets can produce more accurate results comparing with the traditional classifiers, but the outcome greatly depends on the kernel types used, the choice of parameters for the chosen kernel and the method used to generate the SVM.

Chen and Ho (2008) provided an excellent general reference for statistical learning in remote sensing. For linearly not separable cases, the input data were mapped into a high dimensional space, using so-called a kernel function. A training data set of samples, in a d-dimensional feature space $d$, was given by $x_{i}$ with their corresponding class labels:

$$
y_{i}= \pm 1, \Omega=\left\{\left(\mathbf{x}_{i}, y_{i}\right) \mid i \in[1, \ell]\right\}
$$

The linear hyperplane $\mathrm{f}(\mathrm{x})$ was given by the normal vector $\mathrm{w}$ and the bias $\mathbf{b}$, with $|b| /\|w\|$ as a distance between the hyperplane and the origin, where $\|w\|$ was the Euclidean norm of $\mathrm{w}$, given by next.

$$
\mathrm{fl}(\mathrm{x})=\mathrm{wx}+\mathrm{b}
$$

The margin maximization leaded to the following optimization problem:

$$
\min \left[\frac{w^{2}}{2}+C \sum_{i=1}^{\ell} \zeta_{i}\right]
$$

where $\zeta_{\mathrm{i}}$ : denoted the slack variables, and

$\mathrm{C}$ : the regularization parameter, which is used to penalize training errors.

The SVM decision function for a non-linear separable case was described by

$$
f_{n}(x)=\left(\sum_{i=1}^{\ell} \alpha_{i} y_{i} k\left(x_{i}, x_{j}\right)+b\right)
$$

where $\alpha_{i}$ was Lagrange multipliers.

The kernel function $\mathrm{k}\left(\mathrm{x}_{\mathrm{i}}, \mathrm{x}_{\mathrm{j}}\right)$ performed a mapping operation and enabled us to work within the newly transformed feature space, only knowing the kernel function. A common kernel function in remote sensing applications was the Gaussian radial basis function $(\mathrm{RBF})$, defined by

$$
k\left(x_{i}, x_{j}\right)=\exp \left[-\gamma\left\|x_{i}-x_{j}\right\|^{2}\right]
$$

The training of an SVM classifier required the adequate definition of the kernel parameter $\gamma$ and the regularization parameter $\mathrm{C}$. The constant $\mathrm{C}$ was used as a penalty for training samples that are located on the wrong side of the hyperplane. It controlled the shape of the solution. Thus, it affected the generalization capability of the SVM. However, the use of inadequate parameter values might result in a less accurate classification. Often the kernel parameters were determined by a grid-search, using n-fold cross validation. Potential combinations of $\mathrm{C}$ and $\gamma$ were tested in a user-defined range and the best 
combinations for $\mathrm{C}$ and $\gamma$ were selected based on the results of the cross validation.

\section{Image filtering}

Classified images often manifested a salt-and-pepper appearance due to the inherent spectral variability encountered by a classification when applied on a pixel-by-pixel basis. Therefore, it was desirable to "smooth" or "filter" the classified output. The median filtering in Convolution and Morphology tools in ENVI was used for post classification filtering by using a kernel size of $3 \times 3$. The median filtering to smooth an image, while preserving edges larger than the kernel dimensions in removing salt and pepper noise or speckle by replacing each center pixel with the median value within the neighborhood specified by the filter size (Castro \& Donoho, 2009).

\section{Accuracy assessment}

In a statistical context, accuracy regarded to the degree of correctness of a classified map, and it is comprised of bias and precision. A thematic map derived with classification may be considered as accurate if it provided an unbiased representation of the land use and land cover in the region it portrays. Therefore, classification accuracy means the degree to which the derived image classification agrees with reality or conforms to the truth at that particular location and epoch. The accuracy check was done by comparing the classification result with reference data that were believed to reflect the true land cover accurately. In this work, user's, producer's, and overall accuracies together with kappa statistics were derived from the error matrix. The producer's accuracy referred the fraction of correctly classified pixels with regards to all pixels of that ground truth class. The user's accuracy, referred to the reliability of classes in classified images. The kappa statistic incorporated the diagonal elements of the classification error matrix, and represented agreement obtained after the elimination of the proportion of agreement that could have occurred by chance. According to Landis \& Koch (1977), Kappa values were grouped into several categories. Values less than zero (0) indicated no agreement, 0-0.20 were regarded as slight agreement, $0.21-0.40$ were considered fair, 0.41-0.60 were considered moderate, $0.61-0.80$ were substantial, and 0.81-1.00 represented an almost perfect agreement.

\subsubsection{Change detection}

The post-classification change detection algorithm was used to determine the change in mangrove from the three different classified images. It was comprised of comparative analysis of independently produced classification maps on different dates, via a mathematical combination of pixel by pixel. The output of this algorithm was in the form of a matrix showing the initial parameter values of different land covers on the columns, and their final state parameters along the rows, together with their respective spatial representation images. The procedure was carried out at two different intervals, for example, the change that occurred during 1988-2001, 2001-2014, and finally from 1988 to 2014. This was perhaps the most common approach to change detection (Jensen, 1996). It was successfully used by many researchers.

\section{RESULTS}

The classification results presented two major classes (mangrove and non-mangrove) mapped. Spatial distribution showed that mangrove areas were concentrated in the coastal estuaries, rivers where interlinked between land and the sea. The real distribution of mangrove in two study sites were mapped and presented the areas of mangrove regeneration and degradation. Mangrove change analysis was performed using mangrove map results in 1988, 2001 and 2014.

\subsection{The West and Central Africa}

\subsubsection{Spatial distribution of mangrove and accuracy assessment}

Spatiotemporal distribution of mangrove showed for three particular years of 1988, 2001 and 2014 in Figure 5.
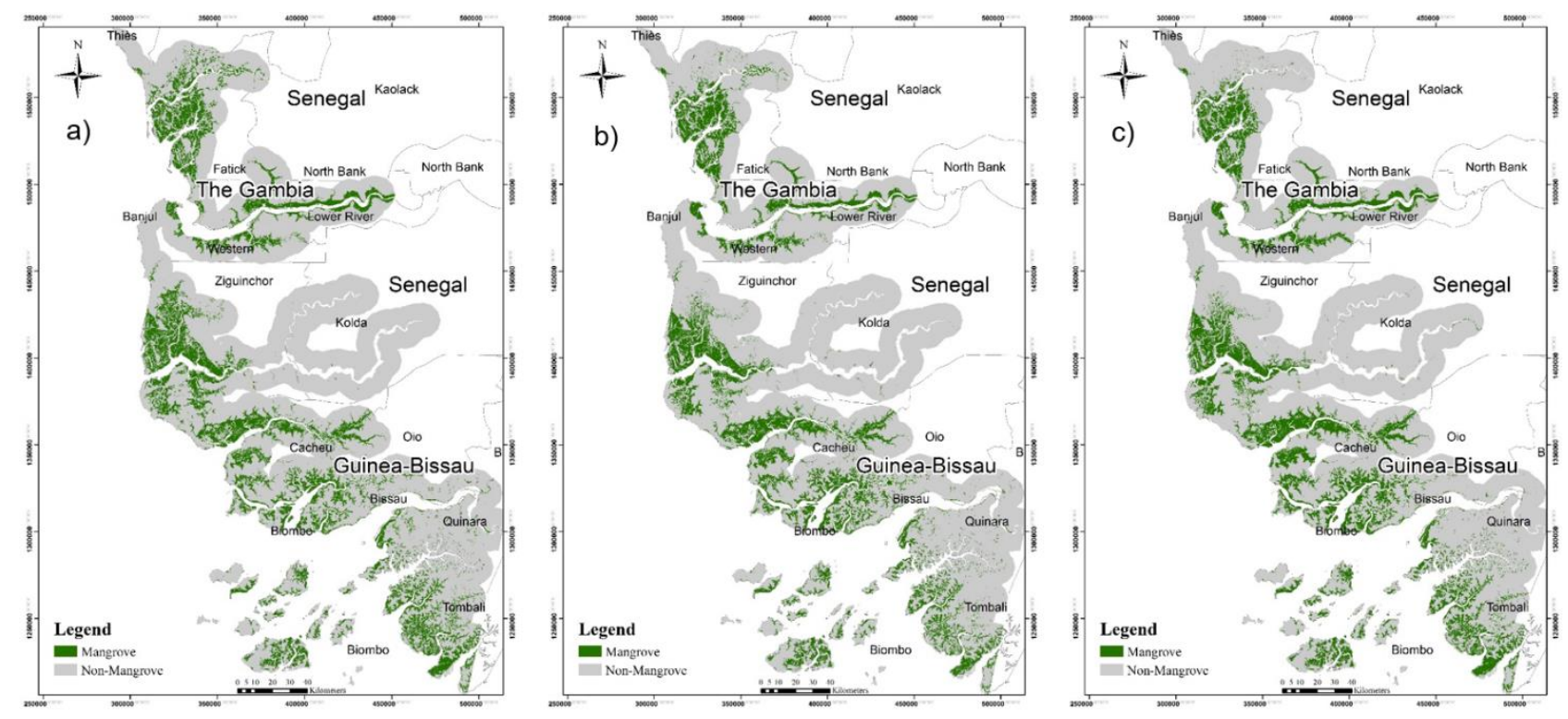

Figure 5. Spatial distriution of mangrove in the West and Central Africa derived from Landsat imagery in a) 1988, b) 2001, and c) 2014. 
Mangrove was generally located in the coastlines, rivers bank, and estuaries. The mangrove was more concentrated in the western of the area, especially in the upper part of the region (northwest). This area was strictly managed by the local governments as natural reserves for biodiversity conservation. Mangrove in the lower part was relatively fragmented due to the development of cultivation land. The study area showed significant rates of mangrove loss in the upstream parts of the rivers, largely due to overexploitation and conversion to agriculture and other uses, and to sedimentation and hydrological changes.

The result of classified maps in 1988, 2001, and 2014 showed that the total mangrove areas were $5,506.4 \mathrm{~km}^{2}(18.7 \%), 5,505.1$ $\mathrm{km}^{2}(18.7 \%)$, and $5,186.0 \mathrm{~km}^{2}(17.6 \%)$, while the non-mangrove area were $23,915.7 \mathrm{~km}^{2}(81.3 \%), 23,917.1 \mathrm{~km}^{2}(81.3 \%)$, and $24,236.1 \mathrm{~km}^{2}(82.4 \%)$, respectively.

For accuracy assessment, random points were generated by using reference map $(10,000$ points for mangrove and 10,000 points for non-mangrove) to validate with the 2014 classification map. The accuracy check was generated an overall accuracy of $84.1 \%$ with kappa coefficient 0.74 . Of 10,000 pixels checked to measure the accuracy each class, the non-mangrove forest class had a higher producer accuracy level (96.7\%). The producer accuracy of the mangrove forest class was $73.54 \%$, which included a corollary omission error of $26.46 \%$.

\subsubsection{Mangrove change detection}

Mangrove has been subjected to enormous pressures and threats within the past three decades. The loss of mangrove from 1988 to 2014 was approximately $16.9 \%$, while only $2.5 \%$ was recovered or newly planted at the same time. The conversion of mangrove to non-mangrove was sharply reduced during the period 2001-2014, when approximately $9.8 \%$ of the mangrove changed to non-mangrove. Mangrove recovered in the region about $3.4 \%$.
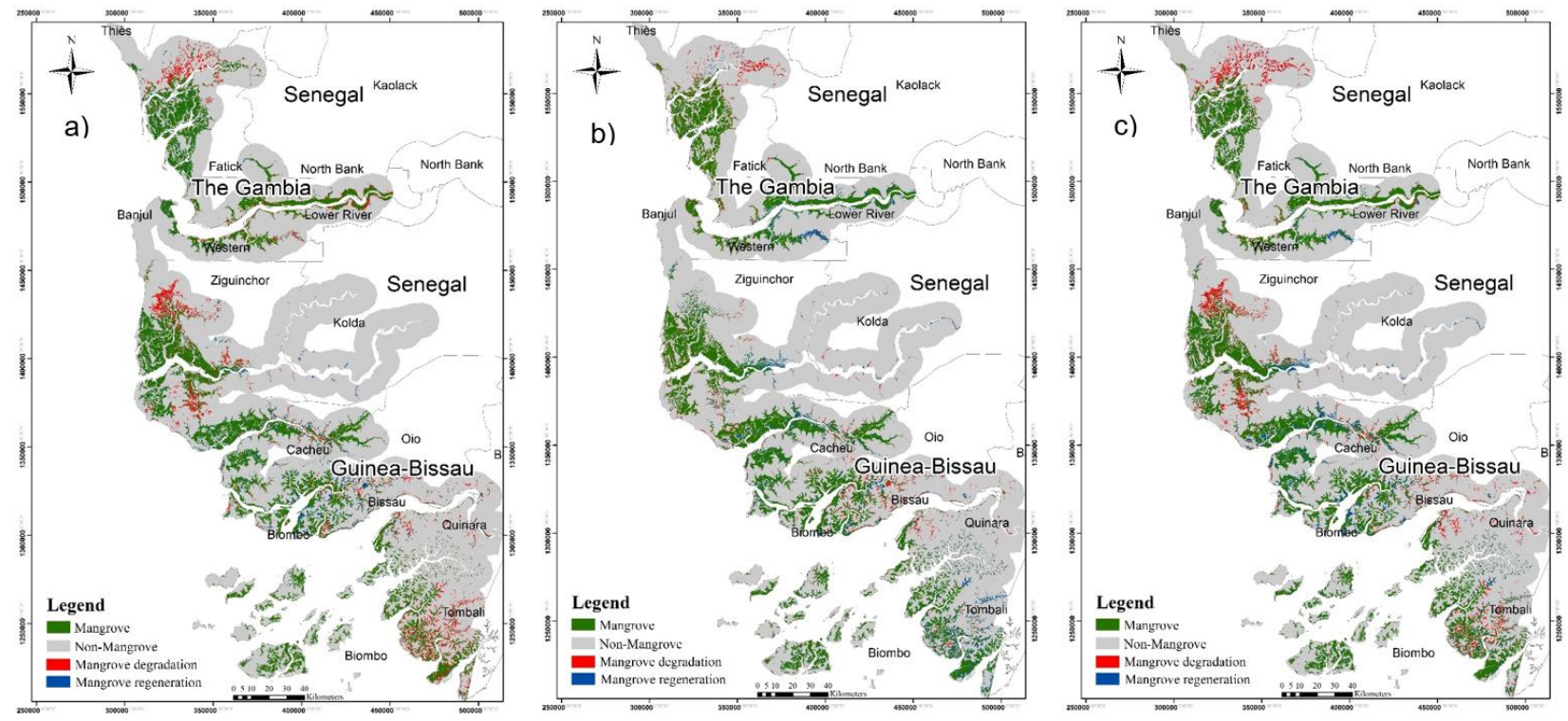

Figure 6. Changes in mangrove forests in the study area from 1988 to 2014. a) Changes in 1988-2001, b) Changes in 2001-2014, and c) Changes in 1988-2014.

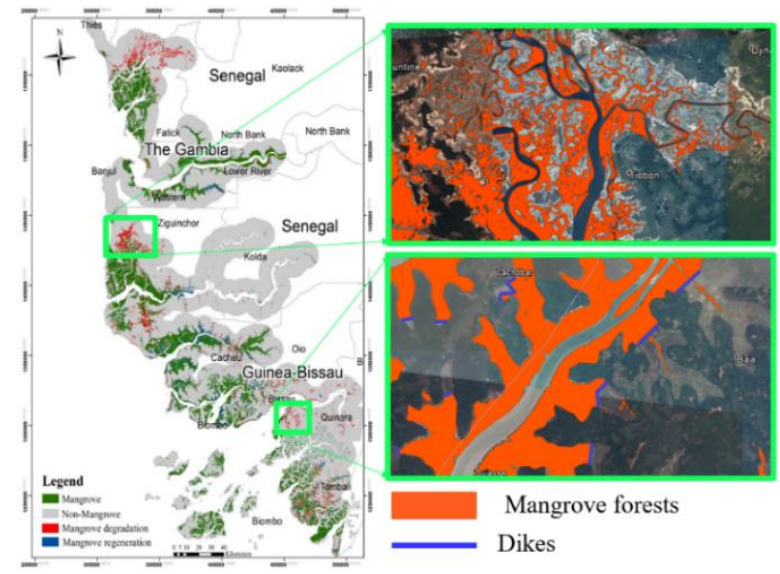

Figure 7. Mangrove deforestation based on native communities' activities and drought and salt intrusions.

These threat factors appeared to be regional from natural to human-made factors with mangrove in the upper Western coasts, and exploitation activities along the coast were also increasingly threatening these mangroves (Figure 7).

\subsection{Southeast Asia - Sundarbans delta}

\subsubsection{Mangrove extraction results in the Sundarbans delta and accuracy check}

According to the classification maps and statistical data derived by Figure 8 , the real estimate of mangrove in the study were $6,635 \mathrm{~km}^{2}(75.0 \%), 5,993 \mathrm{~km}^{2}(68.7 \%)$, and $5,883 \mathrm{~km}^{2}(69.3 \%)$ in 1989,2001 , and 2014, respectively, while $2,212 \mathrm{~km}^{2}(25.0 \%)$, $2,727 \mathrm{~km}^{2}(31.3 \%)$, and $2,613(30.8 \%)$ of non-mangrove were estimated in 1989, 2001, and 2014. The rate of change was not uniform. These changes were non-significant in the context of errors associated with classification and the dynamic nature of mangrove ecosystems. These changes were well within the error margin. For example, selected areas in flooded areas, barren land, and water bodies could easily be misclassified from one class to another. 


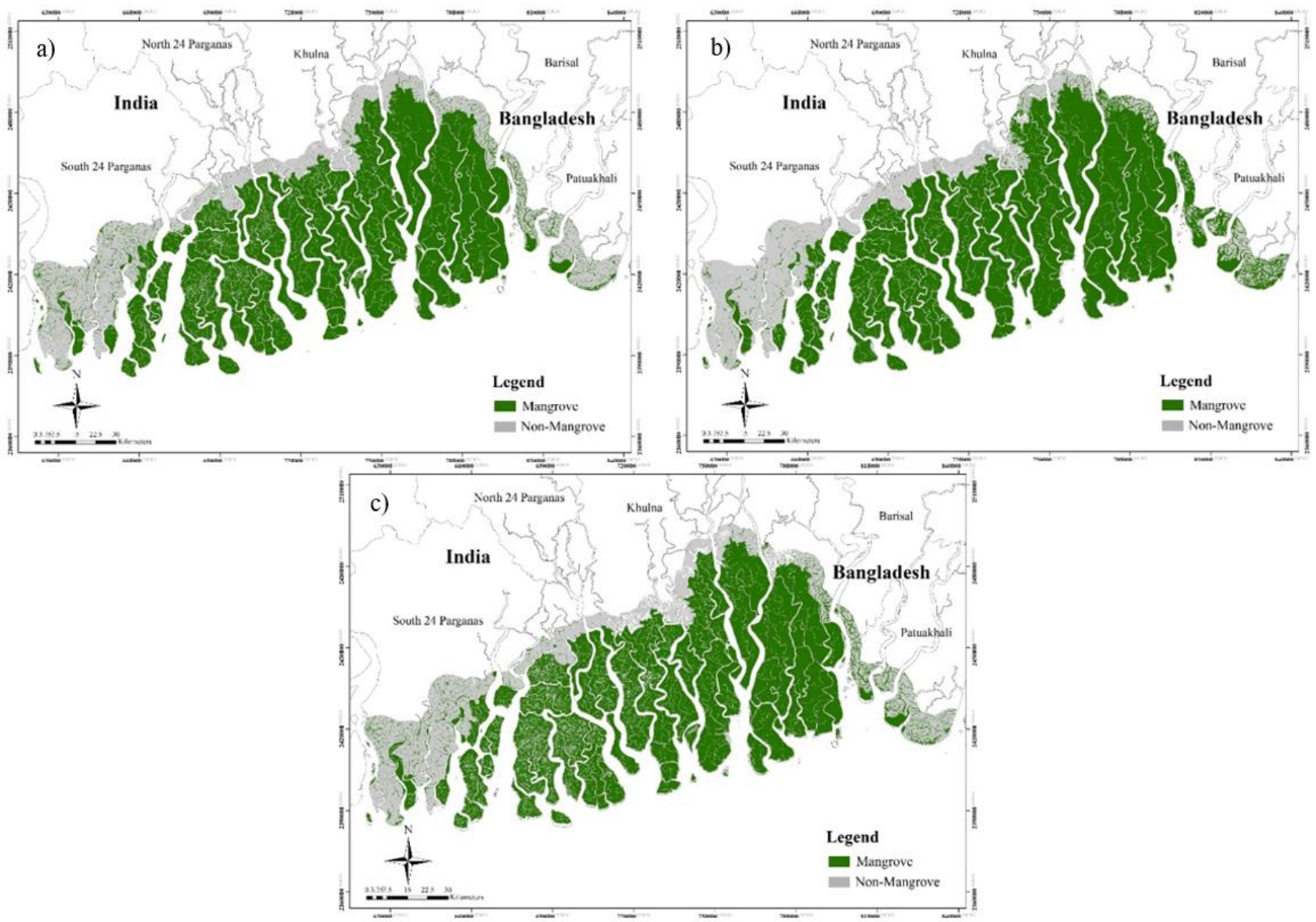

Figure 8. Spatial distriution of mangrove forest in a) 1988, b) 2001, and c) 2014 in the Sundarbans delta derived from Landsat imagery.

Table 2 presented the error matrix comparing the ground reference map with 2014 classification map. The table showed that the overall accuracy was $87.0 \%$ and the Kappa coefficient was 0.73 . The result explanted that mangrove class had a higher producer accuracy level $(91.0 \%)$. The producer accuracy of the non-mangrove class was $76.0 \%$, which included a corollary omission error of $24.0 \%$.

\begin{tabular}{|l|r|r|r|}
\hline \multirow{2}{*}{ Reference data } & \multicolumn{3}{|c|}{ Classification result (2014) } \\
\cline { 2 - 4 } & Mangrove & Non-Mangrove & Total \\
\hline Mangrove & 881 & 100 & 981 \\
\hline Non-Mangrove & 84 & 330 & 414 \\
\hline Total & 965 & 430 & 1,395 \\
\hline Producer accuracy & $91 \%$ & $76 \%$ & \\
\hline User accuracy & $89 \%$ & $29 \%$ & \\
\hline Overall accuracy & \multicolumn{3}{|c|}{$87 \%$} \\
\hline Kappa coefficient & \multicolumn{3}{|c|}{0.73} \\
\hline
\end{tabular}

Table 2. The error matrix for classification results in 2014 comparing with ground reference map in 2011 with some modifications by the author.

\subsubsection{Change in mangrove in the Sundarbans delta in three past decades}

The change of mangrove in three periods, 1989-2001, 20012014, and 1989-2014 presented that the overall change of mangrove in the Sundarbans delta dramatically increased by approximately $15.3 \%\left(900 \mathrm{~km}^{2}\right)$ of the total mangrove area from non-mangrove areas and nature effected.
Similar patterns of change were observed $18.9 \%\left(1,253 \mathrm{~km}^{2}\right)$ and $9.4 \%\left(563 \mathrm{~km}^{2}\right)$ from the 1989 to 2001 and from 2001 to 2014 (Table 3), respectively. The classification results presented that more than $90 \%$ of mangrove, and $75 \%$ of non-mangrove areas did not change. But from 2001 to 2014, about $87 \%$ of mangrove did not change. The large change between mangrove and nonmangrove may possibly be due to variation in tidal inundation at the time of satellite data acquisition. Non-mangrove areas were found in the outer periphery in the Sundarbans. Due to aggradation, land continues to be new in the Sundarbans. This process had increased the land and mangrove areas. The most dramatic and undeniable areas of change were found along waterways in the Bay of Bengal, and some inland areas showed evidence of change as well.

\begin{tabular}{|l|l|l|l|l|}
\hline \multirow{2}{*}{ Period } & \multicolumn{2}{|l|}{ Mangroves } & \multicolumn{2}{l|}{ Non-Mangroves } \\
\cline { 2 - 5 } & $\mathrm{km}^{2}$ & $\%$ & $\mathrm{~km}^{2}$ & $\%$ \\
\hline $1988-2001$ & & & & \\
\hline Mangrove & $5,965.54$ & 94.6 & 669.36 & 24.3 \\
\hline Non-Mangrove & 272.37 & 5.4 & $1,939.15$ & 75.7 \\
\hline $2001-2014$ & & & & \\
\hline Mangrove & $5,859.38$ & 87 & 133.24 & 22.4 \\
\hline Non-Mangrove & 787.43 & 13 & $1,940.04$ & 77.6 \\
\hline 1988-2014 & & & & \\
\hline Mangrove & $5,515.58$ & 91.1 & 367.48 & 24.2 \\
\hline Non-Mangrove & 556.59 & 8.9 & $2,056.06$ & 75.8 \\
\hline
\end{tabular}

Table 3. Changes in the three past decades in Sundarbans. 


\section{CONCLUSIONS}

This research successfully applied to extract mangrove forests based on characteristics, singularities, and distribution as well as reflectance values and spectral properties of mangrove forests in the images. The classification results indicated satisfactory agreement with the ground reference data with overall accuracy of $84.1 \%$ and Kappa coefficient of 0.74 in the West and Central Africa and $83.0 \%$ and 0.73 in the Sundarbans, respectively. In the West and Central Africa, the largest conversion of mangrove to non-mangrove was observed during the period of 1988-2001. The loss of mangrove from 1988 to 2014 was approximately $16.9 \%$, while only $2.5 \%$ was recovered or newly planted. Besides, the overall change of mangrove in the Sundarbans delta increased approximately by $15.3 \%\left(900 \mathrm{~km}^{2}\right)$. Similar patterns of change were observed by $18.9 \%\left(1253 \mathrm{~km}^{2}\right)$ and $9.4 \%\left(563 \mathrm{~km}^{2}\right)$ from the 1989 to 2001 and from 2001 to 2014, respectively. These threat factors appeared to be regional in two cases of the study from natural to human-made factors with mangroves in the upper western coasts, from natural factors mainly of drought and salt intrusions, and wood for construction. In addition, these changes were mainly attributed to the development of aquaculture. Shrimp culture was especially identified as a major cause of direct and indirect loss of mangrove ecosystems due to deforestation for pond construction.

\section{REFERENCES}

Berlanga, R., Ruiz, L., 2002. Land use mapping and change detection in the coastal zone of northwest Mexico using remote sensing techniques, Journal of Coastal Research, 18, 514-522.

Blasco, F., Carayon, J.L. \& Aizpuru, M., 2001. World Mangrove Resources. Glomis Electronic Journal.

Chen, C.F., Nguyen, T.S., Chang, N.B., Chen, C.R., Chang, L.Y., Valdez, M., Centeno, G., Thompson, C.A. \& Aceituno, J.1., 2013. Multi-Decadal Mangrove Forest Change Detection and Prediction in Honduras, Central America, with Landsat Imagery and a Markov Chain Model, Journal of Remote Sensing, 5, 64086426.

Chen, H. \& Ho, P., 2008. Statistical pattern recognition in remote sensing, Pattern Recognition, 41, 2731-2741.

Castro, E. A., \& Donoho, D. L., 2009. Does Median Filtering Truly Preserve Edges Better than Linear Filtering?, The Annals of Statistics 37, no. 3, 1172-1206.

Cideciyan, A.V., Jacobson, S.G., Kemp, C.M., Knighton, R.W., \& Nagel, J.H., 1992. Registration of High-Resolution Images of the Retina, Medical Imaging VI: Image Processing, 1652, 310 322.

Concheddaa, G., Durieuxb, L. \& Mayauxa, P., 2008. An objectbased method for mapping and change analysis in mangrove system, ISPRS Journal of Photogrammetry \& Remote Sensing, 63, 578-589.

Eastman, J.R., 1995. IDRISI for Windows, Version 1.0, Worcester MA: Clark University.

Everitt, J.H., Yang, C., Sriharan, S. \& Judd, F.W., 2008. Using high resolution satellite imagery to map black mangrove on the Texas Gulf Coast, Journal of Coastal Research, 24, 1582-1586.
Giri, C., Pengra, B., Zhu, Z., Singh, A. \& Tieszen, L.L., 2007. Monitoring mangrove forest dynamics of the Sundarbans in Bangladesh and India using multi-temporal satellite data from 1973 to 2000. Journal of Estuarine, Coastal and Shelf Science, $73,91 \mathrm{e} 100$.

Giri, E., Ochieng, L.L., Tieszen, Z., Zhu, A. \& Singh, T., 2011. Global Ecology and Biogeography, Global Ecology and Biogeography, 20, 154-159.

Green, E.P., Mumby, P.J., Edwards, A.J., Clark, C.D. \& Ellis, A.C., 1998. The assessment of mangrove areas using high resolution multispectral airborne imagery, Journal of Coastal Resource, 14, 433-443.

Huang, C., Davis, L.S. and J.R.G., 2002. An assessment of support vector machines for land cover classification, International Journal of Remote Sensing, 23, 725-749.

Islam, M. S., \& Ahmed, R., 2011. Land use change prediction in Dhaka city using GIS aided Markov chain modelling, Journal of Life and Earth Science, 6, 81-89.

Jensen, J. R., 1996. Introductory Digital Image Processing: A Remote Sensing Perspective, 2nd ed. Upper Saddle River, NJ: Prentice Hall.

Landis, J. R., \& Koch, G. G., 1977. The measurement of observer agreement for categorical data, Biometrics, 159-174.

Lugo, A. E. \& Snedaker, S. C., 1974. The ecology of mangroves, Journal of Annual Review of Ecology, Evolution, and Systematics, 5, 39-64.

Vapnik, V., 1979. Estimation of Dependences Based on Empirical Data. Nauka, Moscow, 27, 5165-5184 (in Russian) (English translation: Springer Verlag, New York, 1982).

Vaiphasa, C., Skidmore, A.K. \& de Boer, W.F., 2006. A postclassifier for mangrove mapping using ecological data, ISPRS Journal of Photogrammetry and Remote Sensing, 61, 1-10.

Selvam, V., Ravichandran, K.K., Gnanappazham, L. \& Navamuniyammal, M., 2003. Assessment of community-based restoration of Pichavaram mangrove wetland using remote sensing data, International Journal of Current Science, 85, 794 798.

Seto, K.C., Fragkias, M., 2007. Mangrove conversion and aquaculture development in Vietnam: A remote sensing-based approach for evaluating the Ramsar Convention on Wetlands, Journal of Global Environment Change, 17, 486-500.

Swain, P.H., \& Wacker, A.G., 1971. Comparison of the Divergence and B-Distance in Feature Selection, Laboratory for the Applications of remote Sensing, Purdue University, West Lafayette, 12.

Zhu, G., Blumberg, D.G., 2002. Classification using ASTER data and SVM algorithms: The case study of Beer Sheva, Israel, Remote Sensing of Environment 80 (2), 233-240. 